\title{
SICK BUILDING SYNDROME AMONG HEALTHCARE WORKERS AND HEALTHCARE ASSOCIATES AT OBSERVED GENERAL HOSPITAL IN SLOVENIA
}

\author{
Sedina Kalender-Smajlović1, Mateja Dovjak², Andreja Kukec ${ }^{3}$ \\ ${ }^{1}$ Angela Boškin Faculty of Health Care, Jesenice, Slovenia \\ ${ }^{2}$ Faculty of Civil and Geodetic Engineering, University of Ljubljana, Ljubljana, Slovenia \\ ${ }^{3}$ Faculty of Medicine, University of Ljubljana, Ljubljana, Slovenia
}

\section{SUMMARY}

Objective: The aim of this study was to assess the possible associations between self-perceived sick building syndrome (SBS) symptoms among healthcare workers and healthcare associates and self-perceived parameters of indoor work environment quality.

Methods: The cross-sectional study was conducted from February to April 2019. Validated standardized evaluation tools (MM 040 NA Hospital 2007 and MM 040 NA Office 2007) were used for estimating the prevalence of SBS among observed populations. Chi-square and Mann-Whitney $\mathrm{U}$ tests for assessing possible associations in SBS symptoms between healthcare workers and associates were used.

Results: The response rate was $69.8 \%$. The results showed a lower prevalence of six or more SBS symptoms in healthcare associates $(6.4 \%)$ compared to healthcare workers $(12.0 \%)$. Healthcare workers perceived the most frequent risk factors for SBS to be poor air quality, an inappropriate level of relative humidity, and inappropriate room temperature, while the least frequently self-perceived risk factors were inappropriate lighting and noise levels.

Conclusions: This study represents a platform for further analyses - the identification of health risk factors with environmental monitoring.

Key words: hospitals, healthcare workers, healthcare associates, sick building syndrome, prevalence

Address for correspondence: A. Kukec, University of Ljubljana, Faculty of Medicine, Vrazov Trg 2, SI-1000 Ljubljana, Slovenia. E-mail: andreja. kukec@mf.uni-lj.si

https://doi.org/10.21101/cejph.a6108

\section{INTRODUCTION}

The disease concept of sick building syndrome (SBS) is still unclear (1), although the World Health Organization (2) used the term SBS for the first time to describe situations in which the occupants of a building experience acute health- or comfortrelated effects. SBS is related to numerous heath risk factors. On the basis of systematic review of 313 scientific studies on SBS (3), the health risk factors were identified and classified into six main groups, i.e. physical, chemical, biological, personal, psychosocial, and others. Additionally, for every group, the most important parameters were defined, i.e. 23 in total. Among health risk factors, the most studied are physical and chemical groups, especially related to microclimate conditions and air quality. A few studies highlighted the issue that also psychosocial stress factors and personal factors have and important effect on SBS $(4,5)$. SBS is a condition associated with indoor air quality and comprises symptoms including headache, dizziness, coughing and sneezing, nausea, irritated eyes, irritated throat and nasal mucous membranes, and skin itching and inflammation (6). A hospital environment represents a highly demanding indoor environment, where the needs and demands for defining environmental parameters are based on user specifics, health status, hygiene demands, and specific activities and procedures (7). Moreover, three groups of persons can be identified in a hospital setting: patients, employees, and visitors. Differences in the health status of each of these groups and the diversity of devices that are found in the hospital setting make the hospital microclimate more complex and different from the environment in other public utility buildings (8). Due to their exposure to the complex risk factors in the hospital setting, healthcare workers represent a vulnerable population group. In implementing preventive activities and providing professional health care to patients, healthcare workers are themselves exposed to dangers in the workplace which can jeopardize their health and well-being (9).

A varied prevalence of SBS in healthcare workers was found in previous studies investigating environmental factors in hospitals. According to worldwide epidemiological studies, the evidenced prevalence of SBS in the hospital setting was $21-80 \%(6,10)$, and $30 \%$ in other new and renovated public and residential buildings (11). The observed population in these studies mainly comprised healthcare workers with some healthcare associates included as well. Previous research on SBS in a hospital setting examined the prevalence of SBS-related symptoms and indoor air quality among hospital workers in confined and open spaces (12), in neonatal intensive care units (13), and hospital units (14). Healthcare workers were found to suffer from at least one symptom, mainly from: respiratory mucosal irritation, ocular symptoms, and general 
symptoms (12); fatigue, eye irritation and dry facial skin (14); headache, fatigue and dry hands (6); headache, heaviness in the head, low concentration, and dry skin (15).

Studies conducted among healthcare workers and associates in hospitals established varying frequencies of SBS symptoms. Healthcare workers and other employees in two London teaching hospitals demonstrated signs of SBS, as proven by Kelland (16) on a sample of young nurses and administrative staff $(n=110)$. The cross-sectional study on hospital secretaries (10) showed the prevalence of SBS to be $20.9 \%$.

Considering how important healthcare workers and associates are in providing health care to patients and how important is to maintain their health and safety, it is crucial to design a comprehensive approach of support services. In this approach, the first stage would be to estimate the prevalence of SBS in target groups, while the second one would prepare target recommendations for environmental health policies and strategies. A user-centred design with an emphasis on vulnerable population groups, in our case healthcare workers and associates, plays a crucial role in the environmental health activities for specific hospital settings (7). The building design has to follow the basic principles of bioclimatic design (17), starting from location and climate characteristics (18).

Due to the differences in exposure to risk factors in the hospital environment, a detailed estimate of SBS prevalence among healthcare workers and associates is necessary. According to the existing literature reviews, our study will be the first to estimate the prevalence of SBS symptoms among healthcare workers and associates in a Slovenian general hospital. In addition, we will develop a comprehensive approach for assessing SBS and associated risk factors among the target population in case of general hospital which could, with some modification, be applied also to other hospitals.

\section{MATERIALS AND METHODS}

\section{Subjects}

The cross-sectional study was conducted from February to April 2019. With informed consent, 258 healthcare workers (out of 382) and 78 healthcare associates (out of 99) of a Slovenian general hospital responded to our study (response rate of healthcare workers was $67.5 \%$ and response rate of healthcare associates was $78.7 \%$ ).

\section{Observed Hospital}

In the research, we considered a General Hospital Jesenice building, which includes different specialist hospital treatments, specialist clinic treatment, hospital pharmacy, blood and blood products supply, pathoanatomical work, research and educational work, foreign trade activities for the needs of the institute, and economic activities for the needs of the institute (19). The hospital was built in 1948, net floor area is $18.496,82 \mathrm{~m}^{2}$. The hospital consists of several buildings. Buildings $\mathrm{A}$ and $\mathrm{C}$ have five floors with changing rooms for employees, technical rooms, kitchen, hospital pharmacy, emergency room, two operating rooms, intensive care unit, X-ray, clinics, hospital laboratory, emergency rooms, and classroom. Building A is built of reinforced concrete columns with interior brick walls, the roof is flat, constructed in 1979. Building B has six floors with central sterilization room, boiler room, support services, kitchen, and different departments: dialysis, intensive care unit, clinic, surgery ward, operating room, paediatric clinics, and two paediatric wards. In 1993 and 2010, the department of radiology and support services sections were constructed.

Building $\mathrm{C}$ is made of reinforced concrete bearing walls with interior drywalls, it has a flat roof, constructed in 2015. The loadbearing construction is made of brick with concrete bonds; the building has a gable roof from 1948. Building D has two floors with a gynaecological clinic, security service and dispatcher. The load bearing construction of the building is reinforced concrete. It has interior drywalls, a flat roof was constructed in 2015. Building E has five floors with a day department, physiotherapy, clinics, blood transfusion section (Institute of Transfusion Medicine of the Republic of Slovenia), and a secretariat. The building has a loadbearing construction made of brick with concrete bonds, it has a gable roof from 1963. Building $\mathrm{F}$ has three floors with histopathology laboratory and support services. It is built of a brick with concrete bonds. Building has a gable roof.

The facade of the building is made of interior plaster and a few centimetres polystyrene thermal insulation with a facade plaster, the facade system was made when the paediatric ward (i.e. the entire 4th floor of the main building B) was constructed. The windows were partly replaced during individual renovations (operating wing, sterilization room, partly individual rooms, and doctor's offices). A central ventilation system is not provided.

\section{Data Collection Instrument}

Respondents were asked to complete two questionnaires. Healthcare workers were issued the Indoor Climate at a Hospital/Healthcare Institution Questionnaire (MM 040 NA Hospital 2007) (20), and healthcare associates the Indoor Climate Work Environment-Office (MM 040 NA Office 2007) (21). Both questionnaires collected information on personal factors (education, gender, smoking habits), psychosocial factors (staff category, occupation, working hours, workplace, work pressure, workdays per week, working hours per month, night shifts), environmental factors (draft, room temperature, air quality, noise, light), and typical SBS symptoms, including those related to the eyes (itching, burning or irritation of the eyes), nose (irritated, stuffy or runny nose), throat (hoarse, dry throat, cough), skin (dry or flushed facial skin; scaling/itching scalp or ears; dry, itching, red skin on hands), as well as non-specific, building-related symptoms (tiredness, fatigue, headache, difficulties concentrating, suffering from stress, anger, and dizziness). Data analysis measured the prevalence of specific SBS symptoms reported for five groups of complaints (22). Additional classification of the five groups was performed following the example set in the research by Andersson et al. (23). The first category included a stuffy/runny nose and was termed nasal symptoms. The second category, including dry/irritated eyes, was termed eye-related symptoms. The third category included a hoarse/dry throat and cough; it was termed throat-related symptoms. The fourth category was termed skin-related symptoms and included the symptoms of dry or flushed facial skin, a scaling/ itching scalp or ears, dry, red skin on hands, and itching skin. The fifth category, termed general symptoms, included the symptoms 
of fatigue, heaviness in the head, headache, nausea/dizziness, and difficulties concentrating.

\section{Observed Health Outcomes}

SBS symptoms were assessed with two questions: "Over the last three months, have you had any of the following symptoms (fatigue, headache, etc.)?" Possible answers were: 1 - yes, frequently, 2 - yes, sometimes, 3 - no, never. "Do you believe that this was due to your work environment?" The answers were: 1 - yes, 2 - no, 3 - I do not know. Participants were considered to suffer from SBS if they self-reported having one or more selected symptoms specified in both questionnaires. The observed outcomes included all respondents who answered both questions with 1 - yes; other answers ( 2 - yes, sometimes, 3 - no, never; 2 - no, 3 - I do not know) were not taken into account in computing prevalence. The variables were formulated according to the number of SBS symptoms present and according to the categories of SBS symptoms.

\section{Personal and Work Related Risk Factors}

We included in analysis selected personal (gender, age group) and work related factors (work environment, work conditions, previous/present diseases, work environment in general, temperature conditions, noise, and air quality).

\section{Methods of Analysis}

In addition to basic statistical parameters (frequencies, percentages, means, standard deviation), the Chi-square test and the Mann-Whitney U test were used to compute the prevalence and estimation of differences in SBS symptoms among healthcare workers and healthcare associates working in a Slovenian general hospital. Association between the two nominal variables was tested with the chi-square test or in case of expected frequencies below 5 likelihood ratio Kullback 2Î was used. The level of statistical significance was set at $\mathrm{p} \leq 0.05$ for all statistical tests. The SPSS statistical software for Windows (Version 21.0; IBM Corp.; Armonk, NY, USA) (License: University of Ljubljana, Slovenia) was used as an analysis tool.

\section{RESULTS}

\section{General Characteristics of Participants}

A total of 336 healthcare workers and associates employed in a general hospital in Slovenia were included in the study (of these, 258 were healthcare workers and 78 healthcare associates). The group of healthcare workers included physicians, nursing professionals, midwifery professionals, and other healthcare employees such as physiotherapists, radiographers, occupational therapists, laboratory technicians, and pharmacists (Table 1). The group of healthcare associates included professionals employed in finance and accounting, IT services, purchasing, supply and public procurement, organization and human resources, and planning and analysis services. In addition, we included employees from technical support and maintenance, administrative and legal services, supplies, and senior management of the hospital (Table 2).

The results focus on the differences in the prevalence of SBS symptoms in healthcare workers and healthcare associates employed in a Slovenian general hospital. Data analysis yielded the prevalence of specific SBS symptoms reported for five groups of complaints. Table 3 reveals significant differences in the number of SBS symptoms reported by all healthcare workers in the observed hospital $\left(\chi^{2}=37.698 ; \mathrm{p}=0.014\right)$. Of the total of 258 healthcare workers, $12 \%$ reported six or more SBS symptoms, $19 \%$ reported 2-3 SBS symptoms, and 57.8\% reported 0-1 SBS symptoms. The most frequently self-reported category of SBS symptoms among healthcare workers was general symptoms $(51.9 \%)$, followed by skin-related symptoms (22.5\%), eye-related symptoms (10.1\%), nasal symptoms $(8.1 \%)$, and, finally, throat-related symptoms (6.2\%). The prevalence of nasal symptoms (stuffy/runny nose) was $8.1 \%$ according to the self-report of all healthcare workers. A significant difference between the observed groups of healthcare workers $\left(\chi^{2}=11.407 ; \mathrm{p}=0.012\right)$ was established: most symptoms $(23.1 \%)$ were reported by healthcare workers at the department of paediatrics. The prevalence of skin-related symptoms, such as dry or flushed facial skin, a scaling/itching scalp or ears, dry, red skin on hands, and itching skin, was $22.5 \%$ for all healthcare workers. Our research revealed significant differences between different groups of healthcare workers $\left(\chi^{2}=16.657 ; p=0.020\right)$, with those working at the department of surgery reporting the highest prevalence $(36.8 \%)$. For general SBS-related symptoms, a statistically significant difference was established in their prevalence among the groups of healthcare workers employed at different hospital departments/units $\left(\chi^{2}=14.999 ; p=0.036\right)$, with those employed at the department of surgery reporting them most frequently $(67.6 \%)$, followed by those employed at the non-acute physical rehabilitation services $(63.6 \%)$. No significant differences were established in the prevalence of eye- $\left(\chi^{2}=5.062 ; \mathrm{p}=0.652\right)$ and throat-related symptoms $\left(\chi^{2}=7.589 ; p=0.370\right)$ between groups of healthcare workers employed at different hospital departments/units.

Table 4 shows statistically significant differences in the number of SBS-related symptoms in two groups of healthcare associates: those with a primarily sedentary job and those who occasionally walk or stand in their otherwise sedentary job $\left(\chi^{2}=8.138\right.$; $\mathrm{p}=0.043)$. A total of $66.7 \%$ of healthcare associates reported $0-1$ SBS symptoms (the number was $76.2 \%$ in those with a primarily sedentary job), and $21.8 \%$ reported $2-3$ SBS symptoms (25\% of those who occasionally also walk or stand). Similarly to healthcare workers, healthcare associates also reported general SBS symptoms as the most common complaint (44.9\%); there was no significant difference between the two groups of healthcare associates $\left(\chi^{2}=1.698 ; p=0.194\right)$. Conversely, we found a significant difference in the frequency of skin-related SBS symptoms between the two groups $\left(\chi^{2}=3.478 ; p=0.062\right)$, with healthcare associates who occasionally walk or stand in their otherwise sedentary job reporting more of these symptoms $(22.2 \%)$. This result is not a statistically significant result since $p>0.05$. There were no significant differences between both groups of healthcare associates in the prevalence of nasal symptoms $\left(\chi^{2}=0.039\right.$; $\mathrm{p}=0.844)$, eye- $\left(\chi^{2}=2.257 ; \mathrm{p}=0.133\right)$, and throat-related symptoms $\left(\chi^{2}=1.451 ; \mathrm{p}=0.228\right)$.

In establishing associations between self-perceived SBS symptoms reported by healthcare workers and healthcare associates, 


\begin{tabular}{|c|c|c|c|c|c|c|c|c|c|c|c|c|c|c|c|c|c|}
\hline \multirow{2}{*}{ 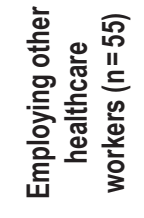 } & $\stackrel{\infty}{\stackrel{\sim}{\sim}}$ & 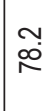 & $\stackrel{\stackrel{P}{F}}{F}$ & $\stackrel{\stackrel{\bigcirc}{\grave{j}}}{\text {. }}$ & $\stackrel{\llcorner}{\circ}$ & 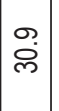 & $\overline{8}$ & $\bar{\sigma}$ & $\stackrel{\infty}{\sim}$ & $\check{8}$ & $\stackrel{\circ}{2}$ & 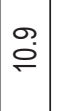 & \begin{tabular}{l|}
$\mathbb{N}^{\infty}$ \\
$\stackrel{0}{ }$
\end{tabular} & ธี & 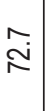 & $\begin{array}{l}\overrightarrow{0} \\
\stackrel{\leftrightarrow}{\circ}\end{array}$ & 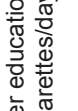 \\
\hline & $\simeq$ & $\mathscr{F}$ & $\mid \begin{array}{l}0 \\
\dot{q}\end{array}$ & $\hat{F}$ & $\overline{\check{\mathbf{T}}}$ & $\approx$ & $\stackrel{\infty}{\infty}$ & 10 & $\simeq$ & $\infty$ & s) & 0 & 으 & $\stackrel{0}{\circ}$ & q & న & 零离 \\
\hline \multirow{2}{*}{ 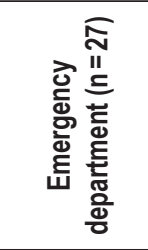 } & $\mid \begin{array}{l}\infty \\
\underset{J}{J}\end{array}$ & ஜֶ & $O \dot{\sigma}$ & 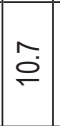 & 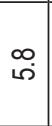 & $\stackrel{\check{F}}{F}$ & $\begin{array}{l}\infty \\
\infty \\
\infty \\
\infty\end{array}$ & $\mid \begin{array}{l}\infty \\
\stackrel{f}{\sim}\end{array}$ & జֶ. & $\because$ & $\ddot{8}$ & $\because$ & $\begin{array}{l}0 \\
\dot{m} \\
\text {. }\end{array}$ & $\stackrel{\sim}{\sim}$ & 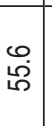 & $\begin{array}{l}\mathscr{D} \\
\infty \\
\infty\end{array}$ & 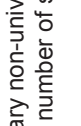 \\
\hline & $\nabla$ & $\approx$ & 离 & $\overline{\check{\sim}}$ & $\overline{0}$ & $m$ & 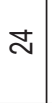 & $\nabla$ & $\approx$ & 0 & $\neq$ & 0 & 으 & $\stackrel{\circ}{\circ}$ & $\stackrel{10}{\circ}$ & 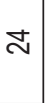 & 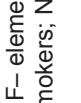 \\
\hline \multirow{2}{*}{ 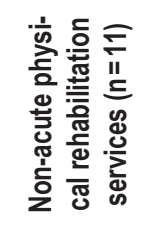 } & $\bar{\sigma}$ & 8 & $\begin{array}{l}\infty \\
\infty \\
\infty\end{array}$ & 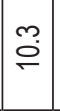 & $\tilde{\omega}$ & $\hat{\mathbf{N}}$ & 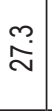 & : & ஓ̊. & $\bar{\sigma}$ & 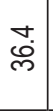 & $\bar{\sigma}$ & 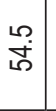 & త్ర & 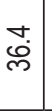 & $\begin{array}{l}\mathscr{0} \\
\ddot{\theta}\end{array}$ & $\sum \Sigma$ \\
\hline & - & 으 & 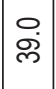 & $\begin{array}{l}\infty \\
\infty \\
\infty\end{array}$ & $\begin{array}{l}0 \\
0 \\
0\end{array}$ & $\infty$ & $m$ & 0 & 으 & - & $\nabla$ & - & 0 & बे & $\nabla$ & $\sim$ & $\frac{\bar{o}}{\bar{g}}$ \\
\hline \multirow{2}{*}{ 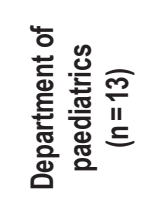 } & 0 & 응 & హ. & $\mathscr{O}$ & $\hat{\sigma}$ & $\mid \begin{array}{l}0 \\
0 \\
0\end{array}$ & 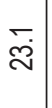 & O. & @̊. & O: & வூ & 잉 & $\begin{array}{l}\infty \\
\stackrel{\rho}{े} \\
\end{array}$ & $\stackrel{\nabla}{\mathrm{N}}$ & \begin{tabular}{l}
$\infty$ \\
\hdashline \\
\hdashline \\
$i$
\end{tabular} & $\underset{\widetilde{\sigma}}{\tilde{\sigma}}$ & 응 \\
\hline & 0 & $\stackrel{m}{\leftrightarrows}$ & \begin{tabular}{|l}
$\infty$ \\
$\infty$ \\
$\infty$
\end{tabular} & $\stackrel{\widehat{o}}{\stackrel{0}{c}}$ & مُ & 은 & $m$ & 0 & $\stackrel{m}{\square}$ & 0 & $\sigma$ & 0 & $\nabla$ & $\begin{array}{l}2 \\
\infty \\
\infty\end{array}$ & $\sim$ & $\cong$ & \\
\hline \multirow{2}{*}{ 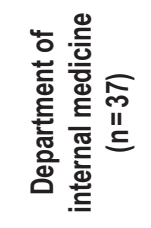 } & 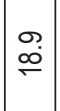 & $\stackrel{\check{\infty}}{-}$ & \begin{tabular}{|l|}
$\infty$ \\
$\infty$
\end{tabular} & $\stackrel{\circ}{\circ}$ & $\begin{array}{l}0 \\
\infty \\
\infty \\
0\end{array}$ & $\stackrel{m}{\sim}$ & 占 & 点 & $\begin{array}{l}0 \\
\dot{\sigma}\end{array}$ & O. & స్రి & $\mid$ & $\stackrel{m}{\sim}$ & छே. & $\underset{\widetilde{d}}{\stackrel{\sim}{*}}$ & $\begin{array}{l}\text { f } \\
\dot{0}\end{array}$ & \\
\hline & $\sim$ & 拊 & 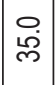 & $\stackrel{\infty}{\stackrel{\sim}{\sim}}$ & $\widehat{\hat{\infty}^{\circ}}$ & o) & $\stackrel{\infty}{\sim}$ & $\sim$ & $\stackrel{m}{m}$ & 0 & $\approx$ & in & $\sigma$ & $\stackrel{\leftrightarrow}{\rightleftarrows}$ & $\simeq$ & 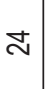 & \\
\hline \multirow{2}{*}{ 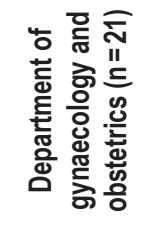 } & 0 & ㅇ. & $\overline{\mathfrak{M}}$ & $\stackrel{\stackrel{\sim}{p}}{\stackrel{p}{\rightleftharpoons}}$ & 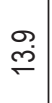 & $\frac{9}{\overline{0}}$ & $\bar{\infty}$ & $\mid$\begin{tabular}{l}
$\infty$ \\
$\leftarrow$ \\
\hdashline
\end{tabular} & ชู & 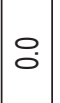 & $\frac{9}{\dot{0}}$ & 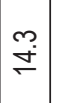 & $\underset{\sim}{\infty}$ & $\stackrel{\infty}{\sim}$ & $\begin{array}{l}0 \\
\dot{F}\end{array}$ & 号 & \\
\hline & 0 & $\bar{N}$ & $\stackrel{\mathscr{\vartheta}}{\dot{\gamma}}$ & $\stackrel{m}{\stackrel{\sim}{\sim}}$ & $\stackrel{\infty}{\rightleftharpoons}$ & $\stackrel{m}{\square}$ & $\infty$ & - & ని & 0 & $\stackrel{m}{\square}$ & $m$ & 10 & $\stackrel{\varphi}{\risingdotseq}$ & 으 & $\stackrel{\circ}{\circ}$ & \\
\hline \multirow{2}{*}{ 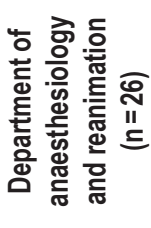 } & $\vec{c}$ & $\begin{array}{l}0 \\
0 \\
i\end{array}$ & $\mathscr{O}$ & $\stackrel{\circ}{\circ}$ & $\begin{array}{l}0 \\
\infty\end{array}$ & 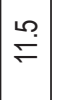 & $\begin{array}{l}\infty \\
\infty \\
\infty \\
\infty \\
\infty\end{array}$ & $\stackrel{\mid}{F}$ & $\begin{array}{l}10 \\
\infty \\
\infty \\
\infty\end{array}$ & $\because$ & 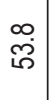 & $\curvearrowright$ & $\begin{array}{l}{\left[\begin{array}{l}0 \\
\infty \\
\infty\end{array}\right.} \\
0\end{array}$ & $\underset{m}{m}$ & 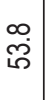 & م. & \\
\hline & 0 & ని & $\mid \begin{array}{l}\infty \\
\infty \\
\infty \\
\infty\end{array}$ & 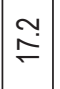 & $\stackrel{m}{\sigma}$ & $m$ & $\approx$ & $m$ & $\approx$ & 0 & 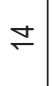 & $\sim$ & 으 & $\begin{array}{l}0 \\
\infty\end{array}$ & 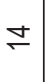 & $\stackrel{0}{\circ}$ & \\
\hline \multirow{2}{*}{ 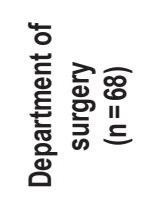 } & $\overline{\mathbf{N}}$ & $\stackrel{\stackrel{g}{N}}{\hat{N}}$ & 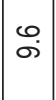 & $\mid$ & $\begin{array}{l}0 \\
0 \\
\infty\end{array}$ & 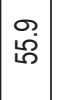 & $\check{f}$ & $\stackrel{\infty}{F}$ & $\begin{array}{l}\infty \\
\infty \\
\infty\end{array}$ & مִ & త్రి & $\stackrel{\sim}{\tilde{m}}$ & $\begin{array}{l}\infty \\
\ddot{m} \\
\tilde{m}\end{array}$ & $\stackrel{\sim}{\forall}$ & 导 & 올 & \\
\hline & $\stackrel{10}{\llcorner}$ & in & 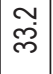 & $\stackrel{\text { م. }}{\rightleftharpoons}$ & 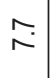 & $\stackrel{\infty}{\infty}$ & ০্ল & $\infty$ & in & - & $\infty$ & 0 & $\approx$ & $\stackrel{\overbrace{}}{\circ}$ & $\hat{m}$ & in & \\
\hline \multirow[b]{4}{*}{ 覀 } & $\underset{⿱}{\stackrel{*}{*}}$ & $\mid \begin{array}{l}\mid \\
\infty \\
\infty\end{array}$ & $\stackrel{+}{0}$ & 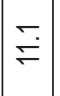 & $\hat{\sigma}$ & 㝎 & 巳̊. & $\left|\begin{array}{l}0 \\
\infty \\
\infty\end{array}\right|$ & 苫 & 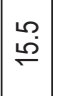 & ஜ் & $\check{\circ}$ & $\stackrel{\infty}{\stackrel{\sim}{\sim}}$ & ¿م. & 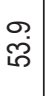 & $\overline{\overrightarrow{0}}$ & \\
\hline & 过 & $\stackrel{m}{\sim}$ & 离 & $\underset{\check{g}}{\check{g}}$ & ब̈. & $\overline{0}$ & $\stackrel{5}{n}$ & $\approx$ & $\stackrel{\leftrightarrow}{\circ}$ & o & 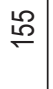 & $\stackrel{\sim}{\sim}$ & 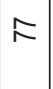 & $\stackrel{\circ}{\circ}$ & $\stackrel{\mathscr{r}}{\square}$ & $\stackrel{\Re}{=}$ & \\
\hline & 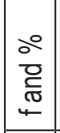 & 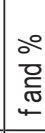 & $\begin{array}{l}0 \\
0 \\
0 \\
\frac{c}{5} \\
\Sigma \\
\Sigma\end{array}$ & 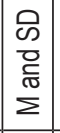 & 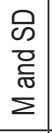 & 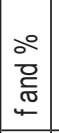 & 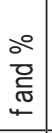 & 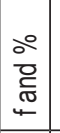 & 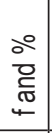 & 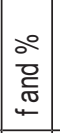 & 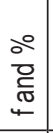 & 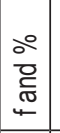 & 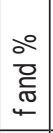 & $\begin{array}{l}0 \\
\text { s. } \\
\text { c } \\
\text { c. } \\
\sum\end{array}$ & $\begin{array}{l}\circ \\
0 \\
0 \\
0 \\
0 \\
\end{array}$ & 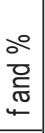 & \\
\hline & $<$ & $\infty$ & 0 & O & ш & 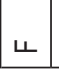 & 0 & \pm & - & $\rightarrow$ & 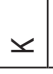 & -1 & $\Sigma$ & $z$ & 0 & a & 8 \\
\hline
\end{tabular}


Table 2. General characteristics of healthcare associates in Slovenian general hospital $(N=78)$

\begin{tabular}{|c|c|c|c|c|c|c|c|}
\hline \multicolumn{4}{|c|}{ Total } & \multicolumn{2}{|c|}{$\begin{array}{l}\text { Employees with a primarily sedentary } \\
\qquad \begin{array}{l}\text { job } \\
(n=42)\end{array}\end{array}$} & \multicolumn{2}{|c|}{$\begin{array}{l}\text { Employees who occasionally walk } \\
\text { or stand in their otherwise sedentary } \\
\text { job }(n=36)\end{array}$} \\
\hline A & $f$ and $\%$ & 13 & 16.7 & 5 & 11.9 & 8 & 22.2 \\
\hline$B$ & $f$ and $\%$ & 65 & 83.3 & 37 & 88.1 & 28 & 77.8 \\
\hline$C$ & Mean and SD & 43.7 & 10.4 & 42.9 & 10.3 & 44.6 & 10.5 \\
\hline$D$ & Mean and SD & 21 & 11.4 & 19 & 11.2 & 23.5 & 11.3 \\
\hline$F$ & Mean and SD & 11.5 & 9.6 & 10 & 8.4 & 13.3 & 10.6 \\
\hline G & $\mathrm{f}$ and $\%$ & 53 & 68.8 & 27 & 64.3 & 26 & 74.3 \\
\hline $\mathrm{H}$ & $f$ and $\%$ & 24 & 31.2 & 15 & 35.7 & 9 & 25.7 \\
\hline I & $f$ and $\%$ & 15 & 21.1 & 7 & 18.4 & 8 & 24.2 \\
\hline$J$ & $f$ and $\%$ & 49 & 69.0 & 28 & 73.7 & 21 & 63.6 \\
\hline K & $f$ and $\%$ & 7 & 9.9 & 3 & 7.9 & 4 & 12.1 \\
\hline$L$ & $f$ and $\%$ & 70 & 89.7 & 37 & 88.1 & 33 & 91.7 \\
\hline M & $f$ and $\%$ & 40 & 51.3 & 24 & 57.1 & 16 & 44.4 \\
\hline $\mathrm{N}$ & $f$ and $\%$ & 10 & 12.8 & 7 & 16.7 & 3 & 8.3 \\
\hline 0 & $f$ and $\%$ & 28 & 35.9 & 11 & 26.2 & 17 & 47.2 \\
\hline$P$ & Mean and SD & 9.3 & 5.0 & 6.8 & 3.2 & 11.2 & 5.4 \\
\hline
\end{tabular}

$\mathrm{n}$ - sample size; $\mathrm{f}$ - frequency; \% - percentage; SD - standard deviation; A - male; $\mathrm{B}$ - female; C - age; $\mathrm{D}$ - total period of employment; $\mathrm{F}$ - period of employmen at current position; $\mathrm{G}$ - elementary non-university higher education; $\mathrm{H}$ - university-level education or higher; I - nursing employees; $\mathrm{J}$ - employees in supporting services; $\mathrm{K}$ - other employees; $\mathrm{L}$ - permanent contract of employment; $\mathrm{M}$ - non-smokers; $\mathrm{N}$ - former smokers; $\mathrm{O}$ - smokers; $\mathrm{P}$ - number of smoked cigarettes/day

the following results were obtained: for the self-perceived number of risk factors in the work environment, a significant difference was found between healthcare workers employed at different departments/units $\left(\chi^{2}=13.648 ; p=0.034\right)$, with those employed at the departments of internal medicine $(\mathrm{n}=37)$ and gynaecology and obstetrics $(n=21)$ reporting the most (mean $=3.35 ; \mathrm{SD}=2.85)$ and the fewest risk factors (mean $=1.14 ; \mathrm{SD}=1.74$ ), respectively. A plurality of healthcare workers $(n=116 ; 45 \%)$ reported at least one SBS risk factor in their work environment, $30.6 \%(n=79)$ reported four or more SBS risk factors, while the fewest $(n=63$; $24.4 \%$ ) reported two to three risk factors in their work environment. Healthcare workers perceived the most frequent SBS related risk factors in their work environment to be poor air quality, inappropriate level of relative humidity, and inappropriate room temperature, and the least frequent to be inappropriate lighting and noise level.

Among the self-perceived parameters of indoor environmental quality, a significant difference was established for air quality in the work environment $\left(\chi^{2}=20.027 ; p=0.006\right)$, with $51.6 \%$ of healthcare workers reporting air quality in the hospital to be inappropriate. Air quality was the most problematic for the group of healthcare workers employed at the non-acute physical rehabilitation services $(81.8 \%)$ and those employed at the department of internal medicine (67.7\%), compared to the least problematic found among those working at the department of gynaecology and obstetrics (28.6\%). No significant differences were established for the self-perceived risk factors in the work environment among healthcare workers employed at different departments/units, namely for the self-perceived inappropriate room temperature $\left(\chi^{2}=12.128 ; \mathrm{p}=0.096\right)$ and inappropriate level of relative humidity $\left(\chi^{2}=9.669 ; p=0.208\right)$. A total of $41.1 \%$ of healthcare workers reported room temperature in the hospital as being inappropriate. This risk factor was most frequent among those employed at the department of internal medicine (56.8\%), followed by those working at the department of anaesthesiology and reanimation and at the department of paediatrics (both 46.2\%), and least frequent among healthcare workers employed at the department of gynaecology and obstetrics (14.3\%). The second most common self-perceived risk factor among healthcare workers was an inappropriate level of humidity $(49.6 \%)$; this risk factor was most frequently reported among those employed at the department of internal medicine ( $64.9 \%)$, followed by those employed at the department of surgery (54.4\%). Inappropriate lighting was a self-perceived risk factor among $34.1 \%$ of hospital healthcare workers. Despite this factor ranking second to last (inappropriate air quality, level of humidity, and room temperature came in first, second, and third, respectively), a significant difference was established for inappropriate lighting according to healthcare workers employed at different departments/units $\left(\chi^{2}=19.756 ; p=0.006\right)$, with those employed at the emergency department most frequently reporting it as a risk factor (59.3\%), followed by those employed at the department of anaesthesiology and reanimation (50\%), and those employed at the department of gynaecology and obstetrics reporting it least frequently $(14.3 \%)$. Despite the fact that not all hospital healthcare workers perceive noise as the most frequent risk factor in the work environment (22.5\%), it turned out to be a frequently self-reported parameter of indoor environment quality in some departments/units. Namely, a significant difference was established among different hospital departments/units for noise as a risk factor $\left(\chi^{2}=14.046 ; p=0.050\right)$. Healthcare workers employed at the emergency department report noise as a risk factor at a rate of $33.6 \%$ - the highest score of all departments - followed by those employed at the department of internal medicine (29.7\%), while those employed at the department of gynaecology and obstetrics do not perceive noise level to be a risk factor at their job $(0 \%)$. 


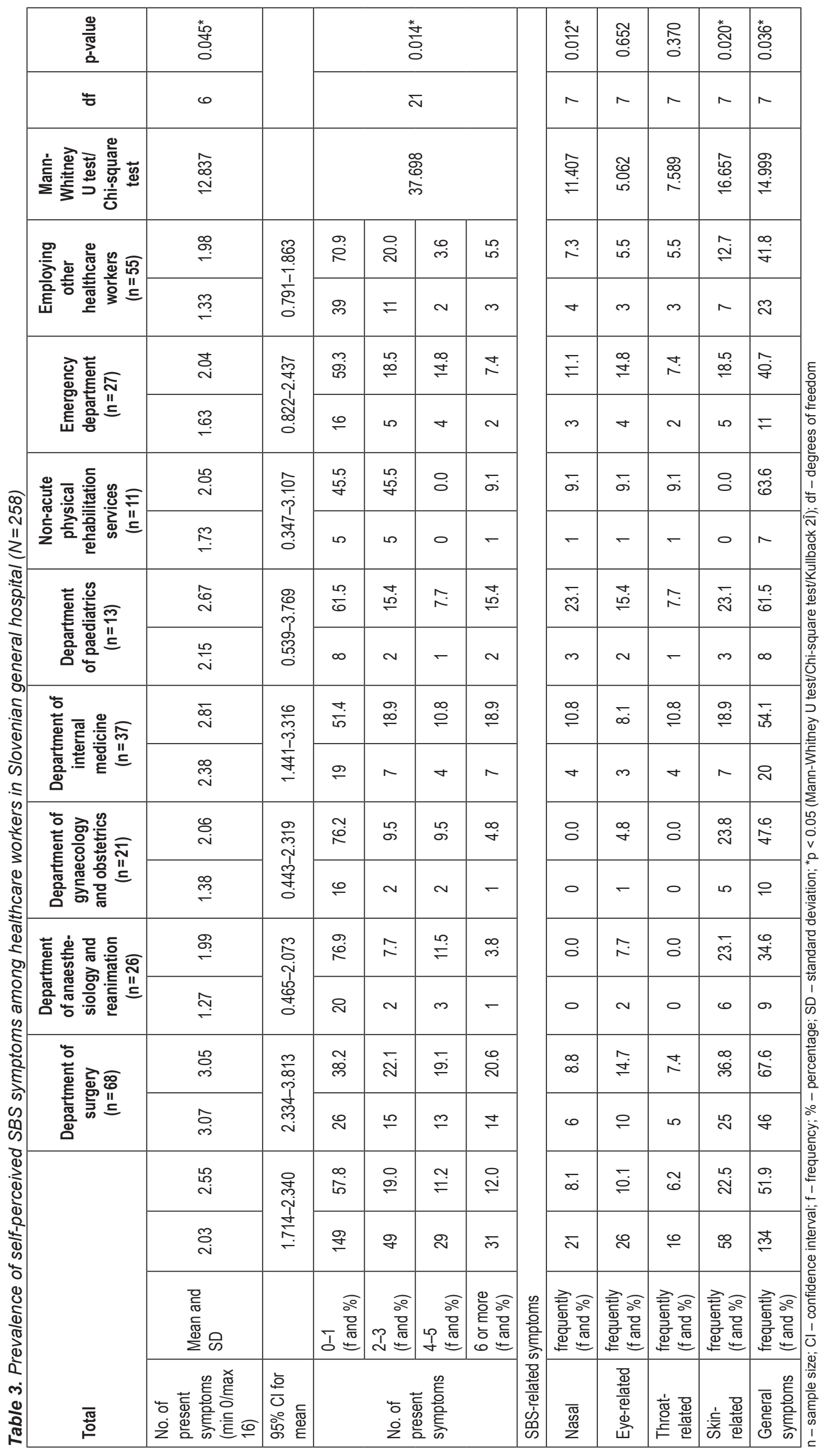


Table 4. Prevalence of self-perceived SBS symptoms among healthcare associates in Slovenian general hospital (N=78)

\begin{tabular}{|c|c|c|c|c|c|c|c|c|c|c|}
\hline \multicolumn{4}{|l|}{ Total } & \multicolumn{2}{|c|}{$\begin{array}{c}\text { Employees with } \\
\text { a primarily sedentary } \\
\text { job } \\
(n=42)\end{array}$} & \multicolumn{2}{|c|}{$\begin{array}{l}\text { Employees who } \\
\text { occasionally walk } \\
\text { or stand in their } \\
\text { otherwise sedentary } \\
\text { job }(n=36)\end{array}$} & \multirow{2}{*}{$\begin{array}{c}\text { Mann-Whitney } \\
\text { U test/Chi- } \\
\text { square test }\end{array}$} & \multirow{2}{*}{$\begin{array}{c}\text { Degrees } \\
\text { of } \\
\text { freedom }\end{array}$} & \multirow{2}{*}{$\begin{array}{c}p \text {-value } \\
0.058\end{array}$} \\
\hline $\begin{array}{l}\text { present } \\
\text { symptoms } \\
\text { (min 0/max 16) }\end{array}$ & Mean and SD & 1.42 & 2.27 & 1.07 & 2.13 & 1.83 & 2.38 & & & \\
\hline $95 \%$ Cl for mean & & \multicolumn{2}{|c|}{$0.584-1.211$} & \multicolumn{2}{|c|}{$0.333-1.191$} & \multicolumn{2}{|c|}{$0.577-1.534$} & & & \\
\hline \multirow{4}{*}{$\begin{array}{l}\text { Number of } \\
\text { present } \\
\text { symptoms }\end{array}$} & $0-1$ (f and \%) & 52 & 66.7 & 32 & 76.2 & 20 & 55.6 & \multirow{4}{*}{8.138} & \multirow{4}{*}{3} & \multirow{4}{*}{$0.043^{*}$} \\
\hline & 2-3 (f and \%) & 17 & 21.8 & 8 & 19.0 & 9 & 25.0 & & & \\
\hline & 4-5 (f and \%) & 4 & 5.1 & 0 & 0.0 & 4 & 11.1 & & & \\
\hline & 6 or more (f and \%) & 5 & 6.4 & 2 & 4.8 & 3 & 8.3 & & & \\
\hline \multicolumn{11}{|c|}{ SBS-related symptoms } \\
\hline Nasal & frequently ( $f$ and $\%$ ) & 6 & 7.7 & 3 & 7.1 & 3 & 8.3 & 0.039 & 1 & 0.844 \\
\hline Eye-related & frequently ( $f$ and \%) & 14 & 17.9 & 5 & 11.9 & 9 & 25.0 & 2.257 & 1 & 0.133 \\
\hline Throat-related & frequently ( $f$ and \%) & 4 & 5.1 & 1 & 2.4 & 3 & 8.3 & 1.451 & 1 & 0.228 \\
\hline Skin-related & frequently ( $f$ and \%) & 11 & 14.3 & 3 & 7.3 & 8 & 22.2 & 3.478 & 1 & 0.062 \\
\hline $\begin{array}{l}\text { General } \\
\text { symptoms }\end{array}$ & frequently ( $f$ and \%) & 35 & 44.9 & 16 & 38.1 & 19 & 52.8 & 1.689 & 1 & 0.194 \\
\hline
\end{tabular}

$\mathrm{n}$ - sample size; $\mathrm{Cl}$ - confidence interval; $\mathrm{f}$ - frequency; \% - percentage; SD - standard deviation; * $p<0.05$ (Mann-Whitney U test/Chi-square test/Kullback 2Î)

Self-perceived parameters of indoor work environment quality were also assessed among healthcare associates according to the number of perceived risk factors present. No significant differences were established between both groups of healthcare associates: those with a primarily sedentary job and those who occasionally walk or stand in their otherwise sedentary job $(\mathrm{p}=0.458)$. We found no significant differences between the two groups for inappropriate room temperature $(p=0.972)$, level of relative humidity $(p=0.448)$, air quality $(p=0.382)$, noise $(p=0.684)$, and lighting $(p=0.490)$. The most frequently self-perceived risk factors among healthcare associates were inappropriate room temperature and air quality ( $47.4 \%$ for both), followed by level of humidity (37.2\%), lighting (29.5\%), and, finally, noise level in the work environment $(24.4 \%)$. No significant differences were established between both groups of healthcare associates on inappropriate room temperature, but $26.2 \%$ of healthcare associates with a primarily sedentary job perceived noise level as a risk factor (compared to $22.2 \%$ of those who occasionally walk or stand in their otherwise sedentary job). Conversely, the second group of healthcare associates more frequently perceived the level of relative humidity $(41.7 \%)$, air quality $(52.8 \%)$, and lighting $(33.3 \%)$ as a risk factor compared to the first group.

\section{DISCUSSION}

Our study evaluated the prevalence of SBS symptoms among healthcare workers and healthcare associates employed in a Slovenian general hospital. The results revealed that $12.0 \%$ of healthcare workers reported experiencing six or more SBS symptoms, while $66.7 \%$ healthcare associates reported experiencing one SBS symptom or none at all. The most frequent complaints among healthcare workers were those categorized as general SBS symptoms (51.9\%), including fatigue, heaviness in the head, headache, nausea/dizziness, and difficulties concentrating. According to hospital departments, the greatest prevalence of general SBS symptoms was reported by healthcare workers employed at the department of surgery and at the non-acute physical rehabilitation services. Skin-related symptoms were reported by $22.5 \%$ of all hospital healthcare workers, most frequently among those employed at the department of surgery. The prevalence of nasal symptoms was not high (8.1\%), but differences existed between the observed groups of healthcare workers, with those working at the department of paediatrics most frequently reporting this complaint (23.1\%). Fewer SBS symptoms were present in research by Chang et al. (12), who found that healthcare workers suffered from at least one symptom at a rate of $84 \%$; the most frequently reported were nasal symptoms, followed by ocular symptoms, fatigue, headache, and facial dryness, with frequency rates of $66 \%, 53 \%, 30 \%, 19 \%$ and $33 \%$, respectively. In addition, a cross-sectional study in a neonatal intensive care unit on a sample of physicians and nurses by Aljeesh et al. (13) showed that about $60 \%$ of healthcare workers suffered from SBS; fatigue and headaches occupied the first and second places among symptoms: $83 \%$ and $76 \%$, respectively. Also Nordström et al. (14) found a high prevalence of SBS in 225 female hospital workers employed at eight hospital units in southern Sweden. The mean value of weekly complaints for fatigue, irritation of the eyes, and dry facial skin was 30\%, 23\%, and 34\%, respectively. Further, Vafaeenasab et al. (6) concluded in their research that the prevalence of SBS among nurses was $86.4 \%$, and there was no association with age, gender, employment history and type of shift work - this was not the case in our study. The most common symptoms among nurses included headache, fatigue and dry hands. 
Our study results revealed a lower prevalence of SBS in healthcare associates compared to healthcare workers employed at the same hospital. A total of $66.7 \%$ of all healthcare associates reported experiencing 0-1 SBS symptoms (the percentage was 76.2 among those with a primarily sedentary job). Further, $21.8 \%$ of healthcare associates reported experiencing 2-3 SBS symptoms ( $25 \%$ of those who occasionally walk or stand in their otherwise sedentary job). Similarly to healthcare workers, healthcare associates also reported general SBS symptoms to be the most frequent (44.9\%); no differences were established between both groups of healthcare associates. Conversely, greater frequency of skin-related SBS symptoms was established among the group of associates who occasionally walk or stand in their otherwise sedentary job. This result is not a statistically significant result since $p>0.05$. No significant differences were established between both groups of healthcare associates in the prevalence of nasal symptoms, eye, and throat-related symptoms. There is little research evidence on the prevalence of SBS symptoms among hospital healthcare associates. The results of a cross-sectional study by Arikan et al. (10) were similar to our findings but they only included administrative workers (secretaries, $\mathrm{n}=177$ ) working at a hospital and found the risk of SBS to be 2.9 times greater for females, 2.8 times greater for those who perceived their work environment as dusty, and 2.6 times higher for respondents who complained of stuffy/bad air, dry air, and an unpleasant odour. According to Kelland (16), staff groups (healthcare workers and administrative staff) in one hospital experienced a higher symptom rate compared to those working in another hospital. This was related to a lower perceived quality of the work environment, characterized by the perception of dryness, heat and low environmental control.

In the second part of the research, we aimed to identify the self-perceived risk factors for SBS in the work environment by healthcare workers and associates in the observed hospital. Our study results showed that, according to all healthcare workers, the most frequent risk factors were poor air quality, an inappropriate level of relative humidity, and inappropriate room temperature, while the least frequent were inappropriate lighting and noise levels. Next, we wanted to establish which risk factors were seen as the most frequent ones according to the department/unit in which healthcare workers worked. Poor air quality was the most frequent risk factor and proved to be a trigger for healthcare workers employed at the non-acute physical rehabilitation services. The second most common self-perceived risk factor was an inappropriate level of humidity, which ranked the highest among healthcare workers employed at the department of internal medicine. This group of employees also perceived inappropriate room temperature as a risk factor. Although inappropriate lighting came in at second to last as a self-perceived risk factor among all healthcare workers, it ranked highest among those employed at the emergency department, followed by those employed at the department of anaesthesiology and reanimation. Healthcare workers employed at the emergency department also reported noise level to be a significant risk factor, followed by those employed at the department of internal medicine. Healthcare workers employed at the department of gynaecology and obstetrics did not perceive air quality, noise level, inappropriate lighting, or room temperature to be a frequent risk factor in their work environment, which could be the result of more effective ventilation and a better layout of working spaces. According to the existing classification $(3,11$,
24, 25), physical factors include environmental parameters of thermal environment, parameters related to the ventilation of buildings, noise level, vibrations, daylight, electromagnetic field, ions, and general design and ergonomics; of these, the thermal environment is among the most investigated risk factors. Previous research results (6) show that poor room ventilation is among physical factors causing SBS among hospital healthcare workers. The level of humidity/air dryness also plays an important role in the prevalence of SBS symptoms among healthcare workers (26), as do artificial ventilation and poor air-conditioning (16). In Finland, Hellgren et al. (27) showed that healthcare workers complain of SBS symptoms more frequently compared to office workers. A survey conducted with a standardized questionnaire and measurements in Swedish hospitals $(n=104)$ showed that air humidification during the heating season may reduce the prevalence of SBS symptoms in colder climates (28). On a sample of 225 Swedish nurses, ocular symptoms were found to be related to stress in the workplace, static electricity, and high noise levels (55 dB A) (14). Similar conclusions were made by Keyvani et al. (15), who assessed the exposure to noise levels, inappropriate/ poor quality lighting, and unpleasant smells. Further, research by Wieslander et al. (29) examined the possible association between the level of relative humidity in the concrete flooring and building framework of four healthcare institutions on the one hand, and the prevalence of SBS in healthcare workers on the other hand to establish a higher rate of ocular and nasal symptoms in healthcare workers employed in more humid buildings. A Palestinian study $(\mathrm{n}=108)$ conducted in Gaza found that more than half of healthcare workers $(60 \%)$ complained of SBS symptoms, a further $83 \%$ complained of tiredness, and $76 \%$ complained of experiencing headaches; of these, almost $85 \%$ believe these symptoms were related to their work environment and $71 \%$ reported that the symptoms subsided when they left the healthcare institution (13). Characteristics of a healthcare institution also influence the frequency of SBS symptoms, as was demonstrated by Canadian research conducted in Halifax on a sample of 297 healthcare workers from five different hospitals (30). In addition, research investigating healthcare settings in 37 Taiwanese hospitals found that hospital departments and pharmacies had poorer air quality (higher levels of carbon dioxide and volatile organic compounds) (31). Our research results showed that, according to healthcare associates, the most commonly perceived risk factors were inappropriate room temperature and poor air quality, followed by an inappropriate level of humidity and inappropriate lighting. Noise levels in the work environment were found to be the least common self-perceived risk factor among healthcare associates. No significant differences existed between healthcare associates with a primarily sedentary job and those who occasionally walk or stand in their otherwise sedentary job for room temperature, level of relative humidity, poor air quality, noise level, and inappropriate lighting.

According to our results of self-perceived SBS symptoms, it is necessary to implement the assessment approach for SBS among the observed population. As stated by Passarelli (32), SBS does not discriminate; it can affect anyone who occupies an area/building. Employers need to be alert to SBS signs, even if these symptoms do not affect everyone in the building/area and not all occupants show visible signs or effects. A literature review revealed no existing system or methodological approach 
for a comprehensive assessment and control of SBS-related risks in the hospital environment (33).

\section{Limitations}

Our study followed well established methods, combination of measurements and questionnaires, followed by statistical analysis. The limitation of our study is that the results were focused on the physical effects and not psychosomatic effects. Psychosocial stress factors and personal factors should be applied in studies on SBS as it was presented in studies by Runeson et al. (4) and Lu et al. (5).

Especially in buildings where the nature of work is such that employees are subject to higher levels of stress (e.g., health care facilities, hospitals), the impact of stress on SBS should be investigated.

The contribution of stress as a stand-alone factor to SBS in hospital is poorly researched. The interaction between stress and other risk factors has also not been investigated. However, in other working environments $(4,5)$ the psychosocial stress factors and personal factors were studied more in detail. The authors proved that the lowest symptom score was found at a relaxed work situation, irrespective of social support. Psychosocial stress factors as well as personal factors such as gender, age, atopy and asthma, and indoor exposures, should be applied in studies on symptoms compatible with SBS (4).

A small number of cases in individual groups by individual departments represent the limitation of our research as well. Due to the presence of different risk factors in individual departments, we wanted to define the differences between them regarding other similar studies, also taking into account the design of targeted measures according to the identified risk factors by individual departments.

In order to assess the importance of environmental, sociodemographic and other risk factors in relation to SBS in the hospital environment, it is necessary to reward statistical analysis with multivariate models in the future. Similar to research done by Runeson et al. (4), where they used multiple logistic regression to identify significant predictors of different types of symptoms, including age, gender, BMI, tobacco smoking, asthma, atopy, educational level, city size, region, work schedule, work hours, number of years in current occupation, and the eight categories in the three-dimensional model.

\section{CONCLUSIONS}

The study is the first example of establishing the prevalence of SBS in the hospital environment in Slovenia conducted among healthcare workers and healthcare associates. Previous studies mainly investigated healthcare workers, mostly nurses. The advantage of our study is that SBS prevalence was established for all hospital employees, including physicians, nursing professionals, midwifery professionals, and other healthcare associates who represent an indispensable part of a multidisciplinary team. We can transfer the method used in this research also to other buildings and environments such as nursing homes, etc. (26, 34-37). Previous studies in the hospital setting mainly included one group of healthcare associates - healthcare administrators - while our study includes all hospital employees in supporting services. We established a higher prevalence of self-perceived SBS symptoms among healthcare workers compared to healthcare associates of the same observed hospital since healthcare workers reported the highest prevalence of general SBS symptoms, followed by skinrelated symptoms, while healthcare associates reported the highest prevalence of general SBS symptoms and ocular symptoms. We also established significant differences among healthcare workers in the prevalence of nasal symptoms (prevalence was the highest at the department of paediatrics), general SBS symptoms and skinrelated symptoms (prevalence was the highest at the department of surgery), while no significant differences were found for the prevalence of eye- and throat-related SBS symptoms. The healthcare workers perceive poor air quality, an inappropriate level of relative humidity, and inappropriate room temperature to be the most frequent risk factors for SBS in their work environment, while the least frequent factors were inappropriate lighting and noise level; healthcare associates reported the most frequent SBS risk factors to be inappropriate room temperature, poor air quality, an inappropriate level of humidity, and inappropriate lighting, while noise level was the least frequent risk factor.

\section{Conflict of Interests}

None declared

\section{Adherence to Ethical Recommendations}

The National Medical Ethics Committee of the Republic of Slovenia granted permission for the study (August 2018; Code Number: 0120135/2018/10). All participants gave informed consent for the research and their anonymity was preserved. The research conforms to the provisions of the Declaration of Helsinki in 1995 (as revised in Edinburg 2000).

\section{Funding}

This research was funded by the Slovenian Research Agency (research core funding P5-0142 Bio-psycho-social context of kinesiology and No. P2-0158, Structural engineering and building physics).

\section{REFERENCES}

1. Saeki Y, Kadonosono K, Uchio E. Clinical and allergological analysis of ocular manifestations of sick building syndrome. Clin Ophthalmol. 2017; 11:517-22.

2. Indoor air pollutants: exposure and health effects. EURO Rep Stud. 1983;(78):1-42.

3. Dovjak M, Kukec A. Creating healthy and sustainable buildings. An assessment of health risk factors. Cham: Springer Open; 2019.

4. Runeson R, Wahlstedt K, Wieslander G, Norbäck D. Personal and psychosocial factors and symptoms compatible with sick building syndrome in the Swedish workforce. Indoor Air. 2006;16(6):445-53.

5. Lu CY, Tsai MC, Muo CH, Kuo YH, Sung FC, Wu CC. Personal, psychosocial and environmental factors related to sick building syndrome in official employees of Taiwan. Int J Environ Res Public Health. 2017;15(1):7. doi: 10.3390/ijerph15010007.

6. Vafaeenasab MR, Morowatisharifabad MA, Taghi Ghaneian M, Hajhosseini M, Ehrampoush MH. Assessment of sick building syndrome and its associating factors among nurses in the educational hospitals of Shahid Sadoughi University of Medical Sciences, Yazd, Iran. Glob J Health Sci. 2014;7(2):247-53.

7. Dovjak M, Shukuya M, Krainer A. User-centred healing-oriented conditions in the design of hospital environments. Int J Environ Res Public Health. 2018;15(10):2140. doi: 10.3390/ijerph15102140.

8. Śmiełowska M, Zabiegala B, Marć M. Indoor air quality in public utility environments - a review. Environ Sci Pollut Res Int. 2017;24(12):1166-76. 
9. Aluko OO, Adebayo AE, Adebisi TF, Ewegbemi MK, Abidoye, AT, Popoola, BF. Knowledge, attitudes and perceptions of occupational hazards and safety practices in Nigerian healthcare workers. BMC Res Notes. 2016;9:71. doi: 10.1186/s13104-016-1880-2.

10. Arikan I, Tekin Ö, Erbas O. Relationship between sick building syndrome and indoor air quality among hospital staff. Med Lav. 2018;109(6):435-43.

11. Dovjak M, Kukec A. Prevention and control of sick building syndrome (SBS). Part 2, Design of a preventive and control strategy to lower the occurrence of SBS. Int J San Eng Res. 2014;8(1):41-55.

12. Chang CJ, Yang HH, Wang YF, Li MS. Prevalence of sick building syndrome-related symptoms among hospital workers in confined and open working spaces. AAQR. 2015;15(6):2378-84.

13. Aljeesh Y, Al Madhoun W, Abu Shamh I, Arcaya M. Assessment of indoor air quality in neonatal intensive care units in government hospitals in Gaza Strip, Palestine. Public Health Res. 2016;6(1):24-30.

14. Nordström K, Norbäck D, Akselsson R. Influence of indoor air quality and personal factors on the sick building syndrome (SBS) in Swedish geriatric hospitals. Occup Environ Med. 1995;52(3):170-6.

15. Keyvani S, Mohammadyan M, Mohamadi S, Etemadinezhad S. Sick building syndrome and its associating factors at a hospital in Kashan, Iran. Iran J Health Sci. 2017;5(2):19-24.

16. Kelland P. Sick building syndrome, working environments and hospital staff. Indoor Built Environ. 1992;1:335-40.

17. Krainer A, Perdan R, Krainer G. Retrofitting of the Slovene Ethnographic Museum. Bauphysik. 2007;29(5):350-65.

18. Pajek L, Košir M. Can building energy performance be predicted by bioclimatic potential analysis? Case study of the Alpine-Adriatic region. Energy Build. 2017;139:160-73

19. Jesenice General Hospital. Public information [Internet]. Jesenice: Jesenice General Hospital; 2018 [cited 2019 Dec 26]. Available from: https:/ www.sb-je.si/o_bolnisnici/informacije_javnega_znacaja/. (In Slovene.)

20. Andersson K. Indoor climate - Hospital/Health Care Establishment [Internet]. Örebro: Örebro University Hospital; 2007 [cited 2018 Mar 15]. Available from: http://www.mmquestionnaire.se/mmq/mm040_care.pdf.

21. Andersson K. Epidemiological approach to indoor air problems. Indoor Air. 1998;8 (Suppl 4):32-9.

22. Commission of the European Communities. Sick building syndrome: practical guide. Environment and quality of life report, no. 4. Luxembourg: Office for Official Publications of the European Communities; 1989

23. Andersson K, Fagerlund I, Norlén U, Nygren M. The association between SBS symptoms and physical and psychosocial environment of school personnel. Proc Indoor Air. 1999;4:360-5.
24. Yassi A, Kjellström T, de Kok T. Basic environmental health. Oxford: Oxford University Press; 2001

25. Kukec A, Dovjak M. Prevention and control of Sick Building Syndrome (SBS). Part 1: Identification of risk factors. Int J San Eng Res. 2014;(8)1:16-40.

26. Li CS, Hsu CW, Tai ML. Indoor pollution and sick building syndrome symptoms among workers in day-care centers. Arch Environ Health. 1997;52(3):200-7.

27. Hellgren UM, Reijula K. Indoor air problems in hospitals: a challenge for occupational health. AAOHN J. 2011;59(3):111-7.

28. Nordström K, Norbäck D, Akselsson R. Effect of air humidification on the sick building syndrome and perceived indoor air quality in hospitals: a four month longitudinal study. Occup Environ Med. 1994;51(10):683-8.

29. Wieslander G, Norbäck D, Nordström K, Wålinder R, Venge P. Nasal and ocular symptoms, tear film stability and biomarkers in nasal lavage, in relation to building-dampness and building design in hospitals. Int Arch Occup Environ Health. 1999;72(7):451-61.

30. Mendelson MB, Catano VM, Kelloway K. The role of stress and social support in Sick Building Syndrome. Work Stress. 2000;14(2):137-55.

31. Jung CC, Wu PC, Tseng CH, Su HJ. Indoor air quality varies with ventilation types and working areas in hospitals. Build Environ. 2015;85:190-5.

32. Passarelli GR. Sick building syndrome: an overview to raise awareness. J Build Apprais. 2009;5:55-66.

33. Dovjak M, Kukec A, Kristl Ž, Košir M, Bilban M, Shukuya M, et al. Integral control of health hazards in hospital environment. Indoor Built Environ. 2013;22(5):776-95.

34. Norbäck D. An update on sick building syndrome. Curr Opin Allergy Clin Immunol. 2009;9(1):55-9.

35. Bentayeb M, Norback D, Bednarek M, Bernard A, Cai G, Cerrai S, et al. Indoor air quality, ventilation and respiratory health in elderly residents living in nursing homes in Europe. Eur Respir J. 2015;45(5):1228-38.

36. Fink R, Eržen I, Medved S, Kastelec D. Experimental research on physiological response of elderly with cardiovascular disease during heat wave period. Indoor Built Environ. 2015;24(4):534-43.

37. Fink R, Eržen I, Medved S. Symptomatic response of the elderly with cardiovascular disease during a heat wave in Slovenia. Cent Eur J Public Health. 2017;25(4):293-8. 